\title{
Acute statin treatment improves recovery after experimental intracerebral hemorrhage
}

\author{
Dongmei Yang ${ }^{1}$, Jianfeng Zhang ${ }^{1}$, Yuxia Han ${ }^{1}$, Elysia James ${ }^{2}$, Michael Chopp ${ }^{3,4}$, Donald M. Seyfried ${ }^{\text {* }}$ \\ ${ }^{1}$ Department of Neurosurgery, Henry Ford Health System, Detroit, USA \\ ${ }^{2}$ Department of Emergency, Henry Ford Health System, Detroit, USA \\ ${ }^{3}$ Department of Neurology, Henry Ford Health System, Detroit, USA \\ ${ }^{4}$ Department of Physics, Oakland University, Rochester, USA \\ Email: ${ }^{*}$ nsdos@neuro.hfh.edu
}

Received 10 January 2013; revised 12 February 2013; accepted 9 March 2013

Copyright (C) 2013 Dongmei Yang et al. This is an open access article distributed under the Creative Commons Attribution License, which permits unrestricted use, distribution, and reproduction in any medium, provided the original work is properly cited.

\begin{abstract}
Background and Purpose: We have previously demonstrated that 2-week treatment of experimental intracerebral hemorrhage (ICH) with a daily dose of 2 $\mathrm{mg} / \mathrm{kg}$ statin starting 24 hours post-injury exerts a neuroprotective effect. The present study extends our previous investigation and tests the effect of acute high-dose (within 24 hours) statin therapy on experimental ICH. Material and Methods: Fifty-six male wistar rats were subjected to ICH by stereotactic injection of $100 \mu \mathrm{l}$ of autologous blood into the striatum. Rats were divided randomly into seven groups: saline control group $(n=8) ; 10,20$ and $40 \mathrm{mg} / \mathrm{kg}$ simvastatin-treated groups $(n=8)$; and 10, 20 and $40 \mathrm{mg} / \mathrm{kg}$ atorvastatin-treated groups $(n=8)$. Simvastatin or atorvastatin were administered orally at 3 and 24 hours after ICH. Neurological functional outcome was evaluated using behavioral tests (mNSS and corner turn test) at multiple time points after ICH. Animals were sacrificed at 28 days after treatment, and histological studies were completed. Results: Acute treatment with simvastatin or atorvastatin at doses of 10 and $20 \mathrm{mg} / \mathrm{kg}$, but not at $40 \mathrm{mg} / \mathrm{kg}$, significantly enhanced recovery of neurological function starting from 2 weeks post-ICH and persisting for up to 4 weeks post ICH. In addition, at doses of $10 \mathrm{mg} / \mathrm{kg}$ and $20 \mathrm{mg} / \mathrm{kg}$, histological evaluations revealed that simvastatin or atorvastatin reduced tissue loss, increased cell proliferation in the subventricular zone and enhanced vascular density and synaptogenesis in the hematoma boundary zone when compared to salinetreated rats. Conclusions: Treatment with simvastatin or atorvastatin at doses of 10 and $20 \mathrm{mg} / \mathrm{kg}$ significantly improves neurological recovery after ad-

"Corresponding author.
\end{abstract}

ministration during the first 24 hours after ICH. Decreased tissue loss, increased cell proliferation and vascularity likely contribute to improved functional recovery in rats treated with statins after $\mathrm{ICH}$.

Keywords: Simvastatin; Atorvastatin; Neurogenesis; Synaptogenesis; Vascular; Intracerebral Hemorrhage

\section{INTRODUCTION}

Intracerebral hemorrhage (ICH) is a devastating form of stroke subtype with high rates of mortality and morbidity $[1,2]$. Current clinical treatment strategies for ICH involve a combination of medical and surgical intervenetions to reduce bleeding and control hypertension and elevated intracranial pressure (ICP). Current standards of care are not sufficient to improve the clinical outcomes of patients who suffer from ICH [3]. To develop a more effective therapy for $\mathrm{ICH}$, recent research efforts have focused on pharmacological enhancement of endogenous neuroplasticity using statins $[4,5]$, bone marrow stromal cells $[6,7]$ and erythropoietin [8].

Statins, which are HMG-CoA reductase inhibitors, are widely prescribed as cholesterol-lowering medications and have also been shown to exert beneficial pleiotropic effects on neurological diseases [4,9-11]. These effects include reduction of neuroinflammation and oxidative stress, improvement of vascular endothelial function, and enhancement of synaptogenesis [11-14]. Emerging evidence, however, has revealed that high-dose statins may have a negative impact on angiogenesis, an important tissue repair mechanism in the neurodegenerative diseases $[15,16]$. Moreover, in patients with a prior history of stroke, aggressive reduction in cholesterol levels with high-dose atorvastatin (SPARCL trial) increased the incidence of ICH [17]. Thus, statins may have a biphasic 
dose-dependent effect, and this requires further study for development of clinical application in the treatment of ICH. We have previously demonstrated that 2 weektreatment with low-dose statins reduces hematoma volume and tissue loss, and improves neurological functional recovery in rats after ICH. The effect of high-dose statins acutely (within 24 hours) in the treatment of ICH remains unknown. Our study was designed to investigate the functional and histological effect of high-dose statin therapy administration acutely after ICH in rats.

\section{MATERIALS AND METHODS}

\subsection{Experimental Model}

All experimental procedures were approved by the Institutional Animal Care and Use Committee at Henry Ford Health System (IACUC \#0814). Adult male Wistar rats ( $n=56$, weighing $270-330 \mathrm{~g}$ ) were subjected to ICH by stereotactic infusion of $100 \mu \mathrm{l}$ of autologous whole blood into the striatum region adjacent to the subventricular zone (SVZ) $[4,18]$. After the ICH, animals were randomly divided into seven groups: 1) saline control group $(n=8)$; 2) $10 \mathrm{mg} / \mathrm{kg}$ simvastatin-treated group $(n=8)$; 3$) 10$ $\mathrm{mg} / \mathrm{kg}$ atorvastatin-treated group $(n=8) ; 4) 20 \mathrm{mg} / \mathrm{kg}$ simvastatin-treated group $(n=8) ; 5) 20 \mathrm{mg} / \mathrm{kg}$ atorvastatin-treated group $(n=8) ; 6) 40 \mathrm{mg} / \mathrm{kg}$ simvastatintreated group $(n=8)$; and 7) $40 \mathrm{mg} / \mathrm{kg}$ atorvastatintreated group $(n=8)$. Simvastatin or atorvastatin or saline was administered orally at 3 and 24 hours [19]. Animals were sacrificed at 28 days after treatment. All rats received daily injections of bromodeoxyuridine (BrdU) 100 $\mathrm{mg} / \mathrm{kg}$ (Sigma, St. Louis, MO) intraperitoneally starting at 24 hours after injury and subsequently for 13 consecutive days.

\subsection{Neurological Functional Studies}

Functional outcome was assessed using a corner turn test [20] and modified neurological severity score (mNSS) [21] at 1, 4, 7, 14, 21 and 28 days after injury. The corner turn test measures long-term functional recovery by counting the number of times that an animal turns toward the nonimpaired, contralateral (left) side when placed in a corner. The non-injured rat randomly turns either left or right and has a number of 5 from ten trials. The mNSS is used to assess neurological functions by a $0-18$ composite score of motor, sensory, balance, and reflex measures, with higher scores implying greater neurological injury.

\subsection{Histology and Immunohistochemistry}

At the end of 4 weeks, the animals were anesthetized and perfused transcardially with PBS, followed by $4 \%$ paraformaldehyde in PBS. Brain tissues were excised, further fixed in 4\% paraformaldehyde and sliced into seven 2$\mathrm{mm}$-thick sections. Each block was processed and em- bedded in paraffin. Four of the adjacent 6-mm-thick sections were cut from each block and were stained with hematoxylin and eosin ( $\mathrm{H} \& \mathrm{E})$.

The brain tissue residing between +0.1 and $0.86 \mathrm{~mm}$ of the bregma on the third block was the most severely injured and therefore the third block was specifically selected for immunostaining. Every 40th coronal section from +0.1 and $0.86 \mathrm{~mm}$ of the bregma was used for immunohistochemical staining with the same antibody. Sections were blocked in a Tris buffered saline solution containing $5 \%$ normal goat serum, $1 \%$ BSA and $0.05 \%$ Tween-20. Sections were then incubated with the primary antibodies for localization of $\operatorname{BrdU}(1: 100$; a marker for proliferation cells), doublecortin (DCX) (1:50; a microtubule-associated protein expressed almost exclusively in immature neurons), synaptophysin (1:1000; a marker for a protein located on synaptic vesicles which contain the neurotransmitters for the labeling of nerve terminals) and EBA (endothelial barrier antigen) (1:1000, a marker for detection of mature vessels). For DAB staining, the sections were incubated with biotinylated $\operatorname{IgG}$ and followed with an avidin-biotin-peroxidase system (ABC Kit, Vector Laboratories, Burlingame, CA). DAB was then used as a sensitive chromogen for light microscopy. For immunofluorescent staining, the sections were incubated with Cy3- and/or FITC-conjugated antibody (1:200; Jackson ImmunoResearch) at room temperature for 2 hours.

After staining with BrdU, DCX, synaptophysin, and EBA, a series of six slides at various levels from the same block were used for semiquantitative measurements. Quantitative measurements of immunostaining were performed by an observer blinded to the individual treatment status of the animals. All slides were digitized under a $\times 20$ objective lens (Nikon, Eclipse 80 i, Melville, NY) using a CoolSNAP color camera (Photometrics, Tucson, AZ) interfaced with a MetaMorph image analysis system (Molecular Devices, Downingtown, PA). BrdU+ cells and DCX+ signals in the dorsal end of the lateral ventricular zone with $100-\mathrm{mm}$ width and $800-\mathrm{mm}$ length were digitized. The synaptophysin immunoreactive area in the perihemorrhagic striatum was measured. Data are presented as percentage of synaptophysin immunoreactive areas in each field divided by the total area in the field. For quantification of vessels, the number of EBA positive vessels was measured in eight fields of view within the hematoma boundary zone area. Data are presented as the density of EBA immunoreactive vessels relative to the area of the hematoma boundary zone.

\section{STATISTICAL ANALYSIS}

Statistical analysis of neurological functional scores, areas of ICH-related tissue loss, and immunohistochemical results were obtained using ANOVA. Data are presented as the mean \pm SEM, and probability value $<$ 0.05 were considered significant. All measurements were 
performed by observers blinded to individual treatments.

\section{RESULTS}

\subsection{Effects of Statins on Neurological Functional Recovery}

As evaluated by both mNSS and corner turn tests, all $\mathrm{ICH}$-induced rats had similar neurological impairments prior to and at one week after ICH (Figure 1). Rats treated with both statins at doses of 10 and $20 \mathrm{mg} / \mathrm{kg}$ showed significant improvements in mNSS and corner turn test starting at 2 weeks, and persisting up to 4 weeks post $\mathrm{ICH}$. However, rats treated with $40-\mathrm{mg} / \mathrm{kg}$ statins showed no significant improvement in neurological function over the control group, assessed by both mNSS and corner turn tests (Figure 1).

\subsection{Effects of Statins on Tissue Loss}

The data for the percentage of striatal tissue loss on the side of hemorrhage is shown in Figure 1. Both the simvastatin- and atorvastatin-treated groups at doses of 10 $\mathrm{mg} / \mathrm{kg}$ and $20 \mathrm{mg} / \mathrm{kg}$ showed significantly reduced tissue loss compared to control at 4 weeks after ICH. The 40$\mathrm{mg} / \mathrm{kg}$ groups showed no significant difference compared to the control group.

\subsection{Effects of Statins on Tissue Repair}

To determine the effects of statins on SVZ cell proliferation, immunostaining for BrdU was performed (Figure 2). Administration of simvastatin and atorvastatin at doses of $10 \mathrm{mg} / \mathrm{kg}$ and $20 \mathrm{mg} / \mathrm{kg}$ significantly increased BrdU-positive cells in the SVZ but not at $40 \mathrm{mg} / \mathrm{kg}$ when compared with saline-treated rats. To determine the effects of statins on neuroblast numbers, immunostaining for DCX was performed (Figure 2). Treatment with simvastatin and atorvastatin at doses of $10 \mathrm{mg} / \mathrm{kg}$ and 20 $\mathrm{mg} / \mathrm{kg}$ but not at $40 \mathrm{mg} / \mathrm{kg}$ increased DCX expression significantly in the SVZ compared with saline-treated rats. Double staining for BrdU-DCX revealed that some DCX-positive cells were BrdU-positive, indicating newly generated neuroblasts in the boundary area around the hematoma.

To investigate the effects of simvastatin and atorvastatin on neurorestoration, histology and immunohistochemistry were performed. Typical immunostainings for synaptophysin in the control group and the treatment group are presented in Figure 3. Quantitative data revealed that treatment with statins at doses of 10 and 20 $\mathrm{mg} / \mathrm{kg}$ but not at $40 \mathrm{mg} / \mathrm{kg}$ significantly increased ( $\mathrm{p}<$ 0.05) synaptophysin expression in the ICH boundary area compared to saline-treated rats, suggesting that simvastatin and atorvastatin enhance synaptogenesis.

To evaluate the effect of statin therapy on vessel proliferation and density, quantitative measurements of EBA staining were performed. When compared to controls, rats treated with 10 and $20 \mathrm{mg} / \mathrm{kg}$ had significantly increased density (vessels $/ \mathrm{mm}^{2}$ ) of EBA-positive vessels in the hematoma boundary zone area $(\mathrm{p}<0.05)$ compared with saline-treated rats (Figure 3). This increase in EBA-positive vessels was not present with the $40-\mathrm{mg} / \mathrm{kg}$ dose. There was no significant difference in all measured parameters (vessel density with EBA staining, synaptic density, local cell proliferation) between the 10- and 20-mg/kg dose.

\section{DISCUSSION}

This study demonstrates a dose-dependent effect of highdose statins administered acutely after experimental $\mathrm{ICH}$. Simvastatin and atorvastatin at doses of 10 and $20 \mathrm{mg} / \mathrm{kg}$ significantly improved neurological function at 4 weeks post-ICH, whereas the higher dose of $40 \mathrm{mg} / \mathrm{kg}$ did not significantly improve neurological function. These findings are consistent with pathologic findings of signifycantly less tissue loss for the lower dose and more tissue loss for the higher dose. The therapeutic benefits observed with statins at $10-$ and $20-\mathrm{mg} / \mathrm{kg}$ doses may partially be attributed to increased cell proliferation, vascularity and synaptogenesis, as demonstrated by histological analysis.

Currently, patients are admitted to the hospital early (within 3 hours) after ICH onset because of the profound ictus. However, Valiente et al. found a higher 30-day
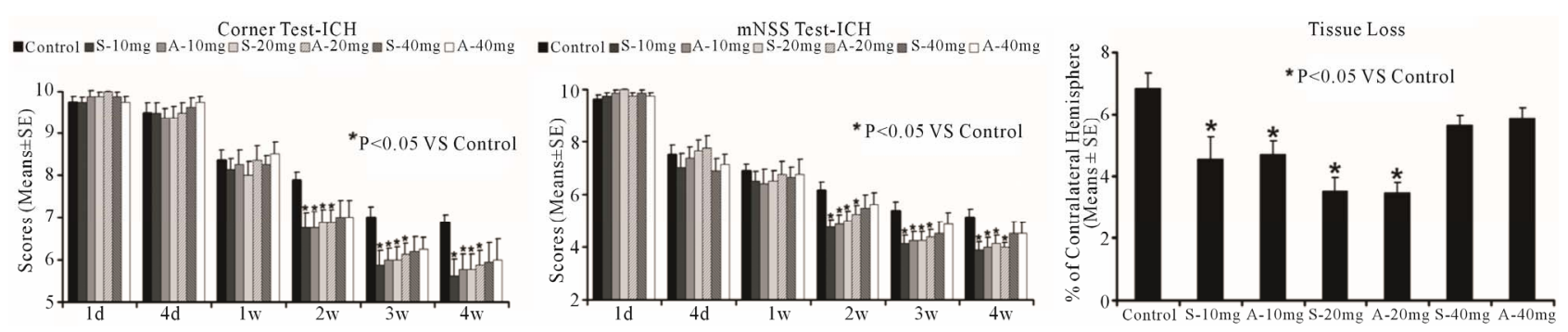

Figure 1. Neurological function tests and striatal tissue loss percentage of the injured side. Quantitative bar graph results of NSS (left panel), corner turn test (middle panel) and striatal tissue loss percentage in the ICH region relative to the contralateral normal region (right panel) of control and six statin-treated groups are presented. Statistical significance is ${ }^{*} \mathrm{P}<0.05$. 

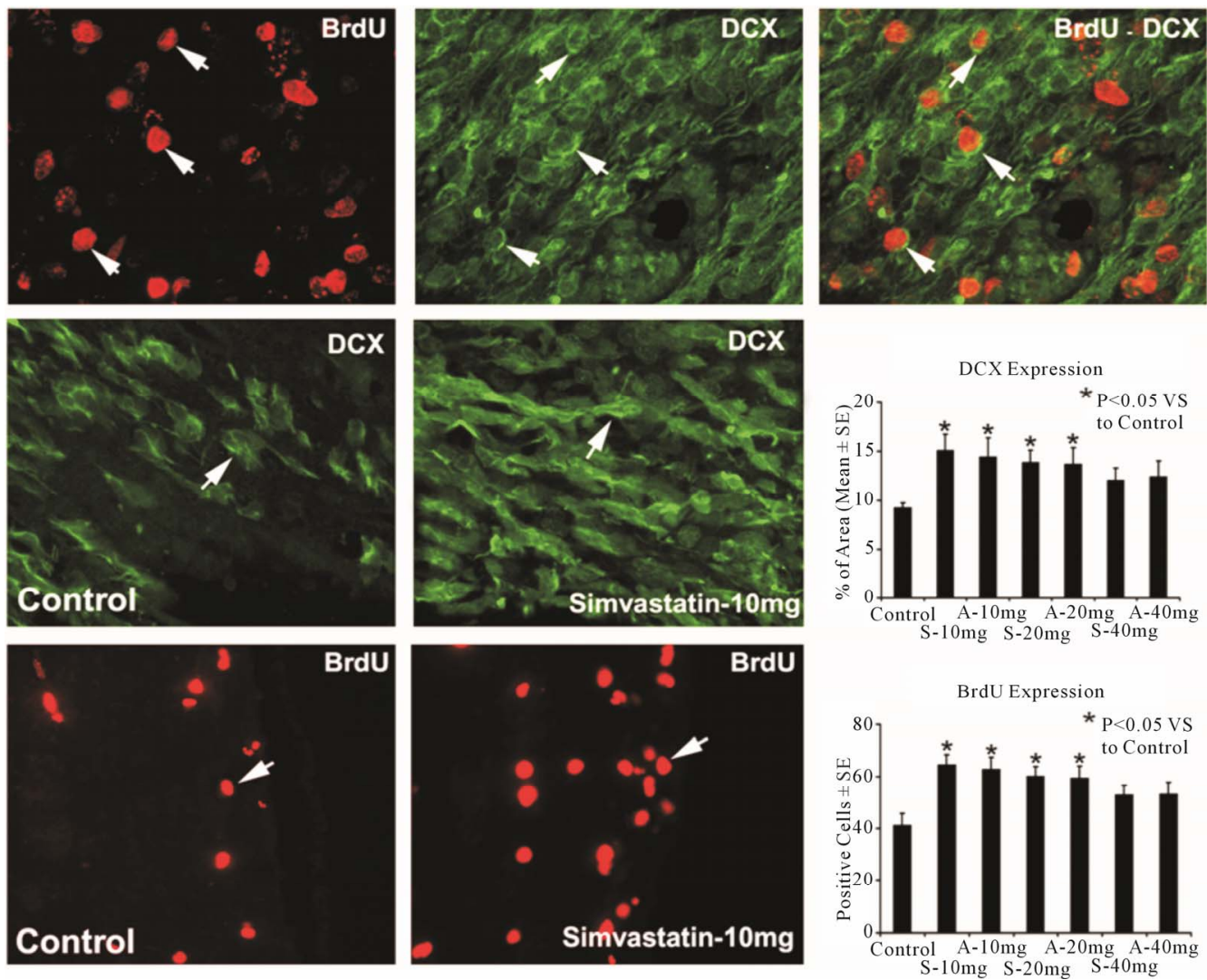

Figure 2. Representative immunostaining and quantitative immunoreactivities of BrdU and DCX from the ICH subventricular zone. Individual images are presented for BrdU (top row) and DCX (top row) that were obtained for the BrdU-DCX double staining methods. The merged image for BrdU-DCX double staining in a subpopulation of cells is presented in the top row. Examples of DCX (middle row) and BrdU (bottom row) stained sections from the subventricular zone of control and simvastatin-treated animals, respectively. Quantitative immunoreactivities of the stainings are shown in the right line.
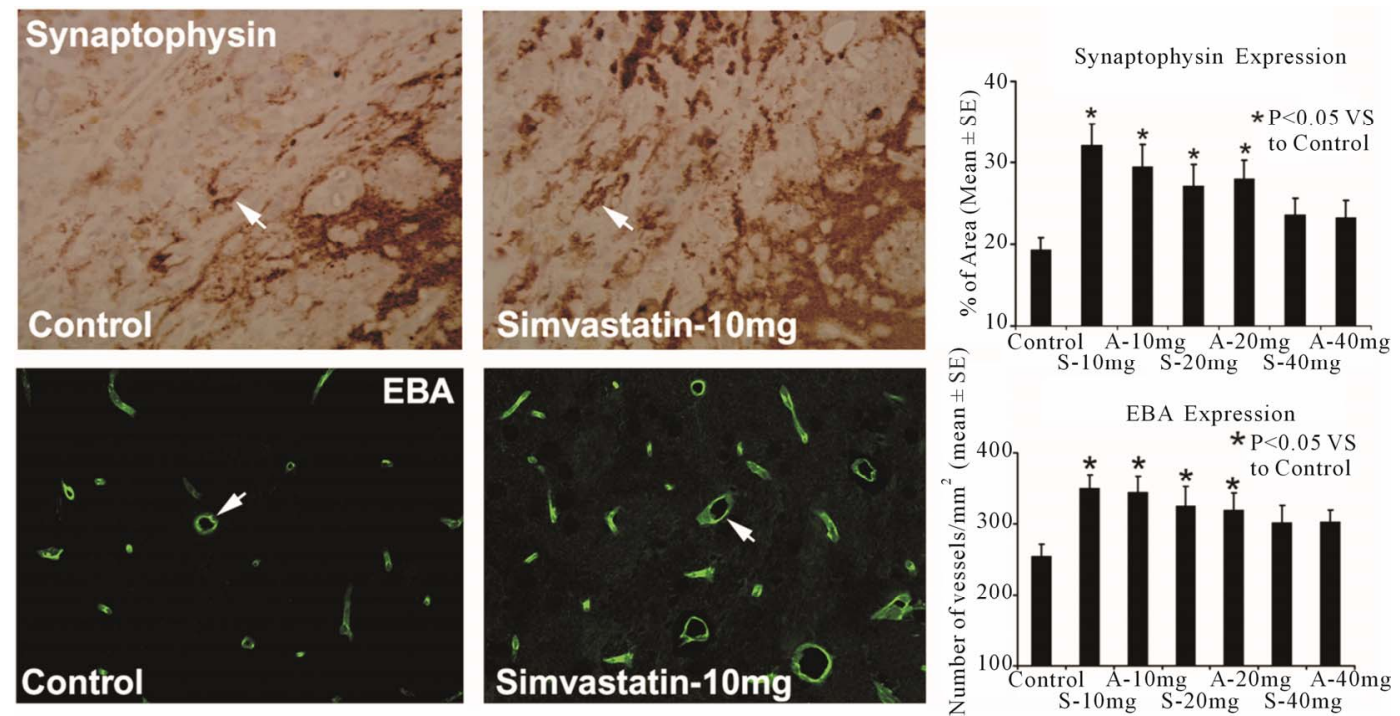

Figure 3. Representative immunostaining and quantitative immunoreactivities of synaptophysin and EBA are shown for the ICH border zone of control and simvastatin-treated rats (left panel and middle panel). Percentage per area of cells positively reacting to synaptophysin and quantitative density (vessels $/ \mathrm{mm}^{2}$ ) of EBA positive vessels in the hematoma boundary zone area for control and statin-treated groups are presented as a bar graph (right panel). 
mortality and a worse outcome for ICH patients admitted within the 3-hour post ictus [22]. Thus, new acute interventions for ICH are needed. Statins have been administered at $3 \mathrm{mg} / \mathrm{kg}$ for one week orally beginning 24 hours after injury to effectively treat $\mathrm{ICH}$ in the experimental ICH model $[4,5,23]$. The early statin treatment after ICH was associated with a 2.5- to 2.8-fold improvement in functional outcomes at 4 weeks, compared with no treatment $[4,5,23]$. The current study focused specifically on the effect of acute 3-hour treatment with high-dose statins after ICH. The results demonstrate that treatment with simvastatin and atorvastatin at doses of 10 and 20 $\mathrm{mg} / \mathrm{kg}$ starting at 3 hours after ICH onset significantly improved neurological functional outcome with a 2.2- to 2.4-fold improvement when compared with no treatment, suggesting that acute treatment of ICH requires a higher dose of the statin to achieve functional benefit. Further study needs to be done to determine whether the favorable outcome after $\mathrm{ICH}$ is related with the onset of statin treatment time.

In our previous study, when atorvastatin was administered for 2 weeks with a low dose of $2 \mathrm{mg} / \mathrm{kg}$, a significant reduction in neurological deficit two to four weeks after the ICH was observed, while a higher dose of 8 $\mathrm{mg} / \mathrm{kg}$ did not improve functional outcome or reduce brain damage [4]. In a stroke model, rats treated with 1 and $3 \mathrm{mg} / \mathrm{kg}$ atorvastatin had significantly improved functional recovery, with no functional benefit detected with $8 \mathrm{mg} / \mathrm{kg}$ atorvastatin, compared with the control treated group [24]. In parallel, the present study showed that simvastatin and atorvastatin treatment initiated at 3 hours post-ICH significantly improved functional neurological outcome acutely at the lower dose, but not at the highest dose. These results suggest that the high-dose statins have adverse effects on functional and tissue recovery, and that the statin-induced adverse effects may be dose-related. The mechanisms for the absence of a therapeutic effect of high-dose statins in this ICH model are not known. Historically, elevated doses of statins may reduce the level of cholesterol linked with an increased rate of hemorrhagic stroke [25-27]. However, the SPARCL study show that the increased risk of ICH with statin therapy was independent of low-density lipoprotein levels [26]. In the current study, the recurrent ICH was not observed in the high-dose statin treated animal during the 4-week survival period. However, several studies indicate that the reduced availability of intracellular levels of cholesterol have an adverse effect on neuronal recovery by interfering with cellular repair and replacement [25-28], which may contribute to the failed functional and tissue effects of high-dose statin. When comparing the plasma levels of atorvastatin administered orally in rats with the plasma levels in humans using an area under-the-curve analysis, doses of $5-18 \mathrm{mg} / \mathrm{kg}$ in rats are equivalent to a dose of $10-80 \mathrm{mg} / \mathrm{kg}$ in humans [19]. In light of these data, high-dose statin therapy should be avoided in $\mathrm{ICH}$ patients until this risk/benefit relationship of high-dose acute statin treatment is better understood.

Several potential mechanisms such as antioxidative, anti-inflammatory, anti-apoptosis, neurotrophism and angiogenesis may contribute to the neuroprotective and neurorestorative effects of statins in experimental $\mathrm{ICH}$ $[4,11,12,29]$. Our data demonstrated that acute treatment with simvastatin and atorvastatin has the ability to reduce tissue loss, improve neurorestoration and improve functional recovery after $\mathrm{ICH}$ in a dose-limited manner. An interesting finding is that treatment with a statin dose of $20 \mathrm{mg} / \mathrm{kg}$ trended towards being slightly more effective at reducing tissue loss compared with a treatment dose of $10 \mathrm{mg} / \mathrm{kg}$. However, the values for neurorestorative markers demonstrated a reverse relationship. This finding suggests that the improved neurological outcome with statin treatment after ICH may be attributed to several potential mechanisms, which individually may have different dose sensitivities, but together promote functional recovery.

There are limitations in the present study. We hypothesized that the effects of low cholesterol in association with the higher dose of statins might be linked to a reduction in functional recovery, but we did not measure plasma cholesterol level. Sironi et al. have demonstrated that simvastatin pretreatment $(20 \mathrm{mg} / \mathrm{kg}$ for 3 days before MCAO) or post-treatment $(20 \mathrm{mg} / \mathrm{kg}$ for 2 days after MCAO) did not affect the plasma cholesterol levels [30]. Further experimentation is needed to measure the statin effect on cholesterol levels in this model, as this study was not specifically designed to evaluate the adverse effects with the use of high-dose statins.

In conclusion, statin treatment at 3- and 24-hours post $\mathrm{ICH}$ is associated with neuronal and functional recovery after experimental ICH. Acute statin therapy 3 and 24 hours after ICH may have significant clinical implications, since most ICH patients would be under medical care at these early time points.

\section{REFERENCES}

[1] Donnan, G.A., Fisher, M., Macleod, M. and Davis, S.M. (2008) Stroke. Lancet, 371, 1612-1623. doi:10.1016/S0140-6736(08)60694-7

[2] Feigin, V.L. (2005) Stroke epidemiology in the developing world. Lancet, 365, 2160-2161. doi:10.1016/S0140-6736(05)66755-4

[3] Bhalla, A. and Hargroves, D. (2008) Does early medical intervention has a role in the management of intracerebral haemorrhage? International Journal of Clinical Practice, 62, 633-641. 
doi:10.1111/j.1742-1241.2007.01691.x

[4] Seyfried, D., Han, Y., Lu, D., Chen, J., Bydon, A. and Chopp, M. (2004) Improvement in neurological outcome after administration of atorvastatin following experimental intracerebral hemorrhage in rats. Journal of Neurosurgery, 101, 104-107. doi:10.3171/jns.2004.101.1.0104

[5] Yang, D., Knight, R.A., Han, Y., Karki, K., Zhang, J., Ding, C., Chopp, M. and Seyfried, D.M. (2011) Vascular recovery promoted by atorvastatin and simvastatin after experimental intracerebral hemorrhage: Magnetic resonance imaging and histological study. Journal of Neurosurgery, 114, 1135-1142. doi:10.3171/2010.7.JNS10163

[6] Seyfried, D., Ding, J., Han, Y., Li, Y., Chen, J. and Chopp, M. (2006) Effects of intravenous administration of human bone marrow stromal cells after intracerebral hemorrhage in rats. Journal of Neurosurgery, 104, 313-318. doi:10.3171/jns.2006.104.2.313

[7] Otero, L., Zurita, M., Bonilla, C., Aguayo, C., Rico, M.A., Rodriguez, A. and Vaquero, J. (2012) Allogeneic bone marrow stromal cell transplantation after cerebral hemorrhage achieves cell transdifferentiation and modulates endogenous neurogenesis. Cytotherapy, 14, 34-44. doi:10.3109/14653249.2011.608349

[8] Cotena, S., Piazza, O. and Tufano, R. (2008) The use of erythtropoietin in cerebral diseases. Panminerva Medica, 50, 185-192.

[9] Lu, D., Qu, C., Goussev, A., Jiang, H., Lu, C., Schallert, T., Mahmood, A., Chen, J., Li, Y. and Chopp, M. (2007) Statins increase neurogenesis in the dentate gyrus, reduce delayed neuronal death in the hippocampal CA3 region, and improve spatial learning in rat after traumatic brain injury. Journal of Neurotrauma, 24, 1132-1146. doi:10.1089/neu.2007.0288

[10] Zhang, L., Chopp, M., Jia, L., Cui, Y., Lu, M. and Zhang, Z.G. (2009) Atorvastatin extends the therapeutic window for tPA to $6 \mathrm{~h}$ after the onset of embolic stroke in rats. Journal of Cerebral Blood Flow \& Metabolism, 29, 18161824. doi:10.1038/jcbfm.2009.105

[11] Jung, K.H., Chu, K., Jeong, S.W., Han, S.Y., Lee, S.T., Kim, J.Y., Kim, M. and Roh, J.K. (2004) HMG-CoA reductase inhibitor, atorvastatin, promotes sensorimotor recovery, suppressing acute inflammatory reaction after experimental intracerebral hemorrhage. Stroke, 35, 17441749. doi:10.1161/01.STR.0000131270.45822.85

[12] Chopp, M. and Li, Y. (2008) Treatment of stroke and intracerebral hemorrhage with cellular and pharmacological restorative therapies. Acta Neurochirurgica Supplement, 105, 79-83. doi:10.1007/978-3-211-09469-3 16

[13] Katsuki, H. Exploring neuroprotective drug therapies for intracerebral hemorrhage. Journal of Pharmacological Sciences, 114, 366-378. doi:10.1254/jphs.10R05CR

[14] Cui, J.J., Wang, D., Gao, F. and Li, Y.R. (2012) Effects of atorvastatin on pathological changes in brain tissue and plasma MMP-9 in rats with intracerebral hemorrhage. Cell Biochemistry and Biophysics, 62, 87-90. doi:10.1007/s12013-011-9264-7

[15] Urbich, C., Dernbach, E., Zeiher, A.M. and Dimmeler, S. (2002) Double-edged role of statins in angiogenesis sig- naling. Circulation Research, 90, 737-744. doi:10.1161/01.RES.0000014081.30867.F8

[16] Fonseca, A.C., Resende, R., Oliveira, C.R. and Pereira, C.M. (2010) Cholesterol and statins in Alzheimer's disease: Current controversies. Experimental Neurology, 223, 282-293. doi:10.1016/j.expneurol.2009.09.013

[17] Goldstein, L.B., Amarenco, P., Szarek, M., Callahan, A., 3rd, Hennerici, M., Sillesen, H., Zivin, J.A. and Welch, K.M. (2008) Hemorrhagic stroke in the stroke prevention by aggressive reduction in cholesterol levels study. $\mathrm{Neu}$ rology, 70, 2364-2370. doi:10.1212/01.wnl.0000296277.63350.77

[18] Yang, S., Song, S., Hua, Y., Nakamura, T., Keep, R.F. and $\mathrm{Xi}, \mathrm{G}$. (2008) Effects of thrombin on neurogenesis after intracerebral hemorrhage. Stroke, 39, 2079-2084. doi:10.1161/STROKEAHA.107.508911

[19] Zhang, L., Zhang, Z.G., Ding, G.L., Jiang, Q., Liu, X., Meng, H., Hozeska, A., Zhang, C., Li, L., Morris, D., Zhang, R.L., Lu, M. and Chopp, M. (2005) Multitargeted effects of statin-enhanced thrombolytic therapy for stroke with recombinant human tissue-type plasminogen activator in the rat. Circulation, 112, 3486-3494. doi:10.1161/CIRCULATIONAHA.104.516757

[20] Zhang, L., Schallert, T., Zhang, Z.G., Jiang, Q., Arniego, P., Li, Q., Lu, M. and Chopp, M. (2002) A test for detecting long-term sensorimotor dysfunction in the mouse after focal cerebral ischemia. Journal of Neuroscience Methods, 117, 207-214. doi:10.1016/S0165-0270(02)00114-0

[21] Chen, J., Li, Y., Wang, L., Zhang, Z., Lu, D., Lu, M. and Chopp, M. (2001) Therapeutic benefit of intravenous administration of bone marrow stromal cells after cerebral ischemia in rats. Stroke, 32, 1005-1011. doi:10.1161/01.STR.32.4.1005

[22] Valiente, R.A., de Miranda-Alves, M.A., Silva, G.S., Gomes, D.L., Brucki, S.M., Rocha, M.S. and Massaro, A.R. (2008) Clinical features associated with early hospital arrival after acute intracerebral hemorrhage: Challenges for new trials. Cerebrovascular Diseases, 26, 404 408. doi:10.1159/000151681

[23] Karki, K., Knight, R.A., Han, Y., Yang, D., Zhang, J., Ledbetter, K.A., Chopp, M. and Seyfried, D.M. (2009) Simvastatin and atorvastatin improve neurological outcome after experimental intracerebral hemorrhage. Stroke, 40, 3384-3389. doi:10.1161/STROKEAHA.108.544395

[24] Amarenco, P., Bogousslavsky, J., Callahan, A., 3rd, Goldstein, L.B., Hennerici, M., Rudolph, A.E., Sillesen, H., Simunovic, L., Szarek, M., Welch, K.M. and Zivin, J.A. (2006) High-dose atorvastatin after stroke or transient ischemic attack. The New England Journal of Medicine, 355, 549-559. doi:10.1056/NEJMoa061894

[25] Karam, J.G., Loney-Hutchinson, L. and McFarlane, S.I. (2008) High-dose atorvastatin after stroke or transient ischemic attack: The Stroke Prevention by Aggressive Reduction in Cholesterol Levels (SPARCL) Investigators. Journal of the CardioMetabolic Syndrome, 3, 68-69. doi:10.1111/j.1559-4572.2008.07967.x

[26] Tirschwell, D.L., Smith, N.L., Heckbert, S.R., Lemaitre, R.N., Longstreth Jr., W.T. and Psaty, B.M. (2004) Asso- 
ciation of cholesterol with stroke risk varies in stroke subtypes and patient subgroups. Neurology, 63, 18681875. doi:10.1212/01.WNL.0000144282.42222.DA

[27] Pfrieger, F.W. (2003) Cholesterol homeostasis and function in neurons of the central nervous system. Cellular and Molecular Life Sciences, 60, 1158-1171.

[28] Indraswari, F., Wang, H., Lei, B., James, M.L., Kernagis, D., Warner, D.S., Dawson, H.N. and Laskowitz, D.T. Statins improve outcome in murine models of intracranial hemorrhage and traumatic brain injury: A translational approach. Journal of Neurotrauma, 29, 1388-1400. doi:10.1089/neu.2011.2117
[29] Sironi, L., Banfi, C., Brioschi, M., Gelosa, P., Guerrini, U., Nobili, E., Gianella, A., Paoletti, R., Tremoli, E. and Cimino, M. (2006) Activation of NF-kB and ERK1/2 after permanent focal ischemia is abolished by simvastatin treatment. Neurobiology of Disease, 22, 445-451. doi:10.1016/j.nbd.2005.12.004

[30] Chen, J., Zhang, Z.G., Li, Y., Wang, Y., Wang, L., Jiang, H., Zhang, C., Lu, M., atakowski, M., Feldkamp, C. and Chipp, M. (2003) Statins induce angiogenesis, neurogenesis, and synaptogenesis after stroke. Annals of Neurology, 53, 743-751. 